\title{
Basic Psychosocial and Biological Contributors to Confirmation Bias
}

\begin{abstract}
Jiaqi Chen
Psychology Department, University of California, San Diego

Correspondence: Jiaqi Chen (jic008@ucsd.edu)

ABSTRACT

Like every natural phenomenon in the world, psychological phenomena take on mechanisms that give rise to them. Without exception, confirmation bias emerges from mechanisms that increase the chances for a person to assert information complacently once a certain threshold is met. The literature on confirmation bias (CB) points to several social, cognitive, and emotional factors in facilitating $\mathrm{CB}$, and more recent work illustrates the neural correlates to some of these factors. However, what is missing in the science on CB are investigations into more "basic" factors that may lead to $\mathrm{CB}$ and constitute the more basic "ingredients" that support and evoke the human mind. The author argues that at least three fundamental factors are uncertainty, valence, and working memory. With these factors in mind, the author summarizes behavioral and neurobiological correlates of $\mathrm{CB}$, mechanisms associated with these behavioral and brain manifestations of $\mathrm{CB}$, and gaps in the literature where a focus on studying uncertainty, valence, and working memory may prove instructive. The author further argues these factors may explain current findings in CB and proposes a model wherein these factors interact mechanistically to give rise to $\mathrm{CB}$.
\end{abstract}

Keywords: cognitive biases, uncertainty, valence, memory, mechanism.

\section{INTRODUCTION}

Most humans contend with being proponents of truth, committed to challenging their implicit biases in hopes of thinking, feeling, and behaving more ethically in the world. Nevertheless, the opposite appears to be truer for human nature: people show tremendous resistance in accepting factual information that contradicts their beliefs and regularly fall prey to a myriad of biases that not only leads to their prejudiced treatment of others, but also the development of psychopathologies like depression and anxiety.

In other words, human minds show profound bias, a disproportionate weight in preference for or against an idea or thing. Critically, bias can be both learned and encoded in human DNA [1]. For instance, individuals quickly learn in their development who is an "outsider" versus an "insider" through socialization [2]. Moreover, research suggests an evolved bias exists to avoid thin, sharp, and pointed objects, which may explain why some individuals develop phobias to snakes and needles [3]. Overall, biases affect our psychology; they influence how people think, feel, and behave. Like most cognitive biases, confirmation bias merits intervention in certain contexts since it can lead to erroneous decision making, frequent discounting of other's counterevidence, and, likely, undergirds many gender- and race-based implicit biases.

Although confirmation bias has been defined in multiple ways (for a review, see [4]), the subject of its definitions revolves around the source of information, the agent attending to that information, and - through mechanisms that is the focus of this paper-the agent's response to that information. Typically, confirmation bias is conceptualized as a mental state in which individuals prioritize confirming information that often preserves one's sense of self- or, more often, to protect their previously held beliefs, either consciously or subconsciously [5]. CB can influence the way individuals perceive conspecifics, cause certain psychological disorders, and reduce openness to experience. In fact, research has shown that people with strong opinions on complex social issues are significantly more likely to consider confirming evidence at face value but view disconfirming evidence more skeptically, leading to an increase in attitude polarization [6]. Although confirmation bias as a mental state involves affective and cognitive components, much of the existing literature treats confirmation bias as a more "cognitive" than "emotional" process [4]. Here, the author seeks to 
balance this perspective by emphasizing both cognitive and affective processes in confirmation bias.

Overall, this paper is divided into three main sections. First, the author introduces two common approaches to investigating or understanding confirmation bias and highlights the corresponding research findings. In addition, this paper briefly describes the factors that the general public believes play a critical role in confirmation bias and theoretically illustrates their convergence in promoting the bias itself. The second section is dedicated to the studied factors, which include valence, uncertainty, and working memory. Lastly, the author outlines possible mechanisms and their directions by which valence, uncertainty, and working memory may exert influence over mental states, thus creating a bias against the aforementioned mental states. The ultimate goal of this paper is to propose an architecture through which confirmation bias is realized neurologically and, therefore, psychologically. It would help the psychology community to develop a more comprehensive understanding of confirmation bias with other significant psychological concepts and provides enlightenment to interested researchers on how to approach, interpret and possibly regulate the phenomenon in the future.

\section{LEVELS OF ANALYSIS}

Based on past literature, confirmation bias has been investigated from at least two levels of analysis: at the person level, confirmation bias is understood as a trait- or state-based phenomenon, and at a mental state level, confirmation bias is recognized as a cognitive or affective response to the immediate environment. In this section, the author discusses each of these approaches using neurological and psychological evidence to explain the bias, and then briefly introduces the relevant constructs that facilitate confirmation bias.

Traits, or persistent and dispositional characteristics of an individual [7], are more easily exploited by an individual's tendency to respond, while the more "statelike" responses are context-dependent [8]. However, it can be difficult to distinguish between "traits" and "states", and this is also true for confirmation bias. A person's tendency toward confirmation bias can be reinforced or suppressed by innate characteristics or external stimuli, depending on the specific situation. There are dopaminergic genes capable of predicting individual differences in susceptibility to confirmation bias [1], which raises the question of whether confirmation bias here is trait-like or state-like, or both. According to this study, the polymorphism in the COMT gene (rs4680), which is associated with prefrontal dopaminergic function, can predict the degree to which participants resist counter-evidence to their propositions. Moreover, brain activity related to firm beliefs is facilitated by the preactivation of frontal cortex (PFC), which modifies the striatal learning process consistent with confirmation bias. These genetic and neuroscientific findings serve as evidence that conceptualizing confirmation bias as "nature vs. nurture" may be too simplistic; instead, it seems that confirmation bias may be a product of "nature" and "nurture".

Similarly, in the presence of confirmation bias, people tend to amplify supportive evidence and without adopting the strength of others' disconfirming opinions, exhibiting reduced neural activity in the medial prefrontal cortex [9], an area implicated in theory of mind. However, it was shown through a quasi-experimental survey that selective pre-election exposure, such as online news, enables information utility (see Table 1 for definition) to override confirmation bias in participants whose favored party is likely to lose [10], which probably results from a less heated mind for winning and more clearance for fair judgments. Moreover, another study found that individuals with a "foreclosed ego identity status", those who are more ready to adopt the attitudes and opinions from others, showed the most confirmation bias than those with an "achievement ego identity status", those who typically search for multiple alternatives instead of adopting ways of living from others [11]. These two pieces of evidence essentially suggest that exposure to relevant evidence induces certain temporary states in which people perceive information in a biased manner. This suggests that the external environment also guides people's decision-making process and is at least partially responsible for the emergence of confirmation bias. Hence, when it is asked whether confirmation bias takes its root as a trait or state in our mind, similar to treating the question of nature versus nurture, it remains unreasonable and insular to exclude either one out of consideration.

As mentioned earlier, confirmation bias can also be understood as either a cognitive or affective state responsive to the environment, not necessarily as a characteristic of an individual (as previously discussed). This dual distinction between cognition and emotion often appears in the scientific literature. While most people view the brain and heart as critical organs, several ancient Greek works of literature emphasize the difference between reason and passion for drawing a dualism between what people think (brain/mind) and what people feel (heart). In psychological science, research on confirmation bias has almost exclusively examined this bias as a cognitive feature of the mind, yet cognition is inextricably entwined with emotion.

Cognitive explanations for confirmation bias underscore the limited capacity in human beings to handle complex tasks in the context of an excessive influx of information. Confirmation bias demonstrates to be a shortcut (heuristic) that the brain uses to solve problems efficiently and conservatively. People are limited in the number of "things" they can think about simultaneously, which forces the brain to be less likely to 
encode alternative hypotheses [5]. On the other hand, though less abundant than cognition, a considerable number of researches on the role of emotion in confirmation bias still reveal the affective influence in information intake and processing. For example, one study states that one who undergoes an anxious state tends to interpret stimuli as more threatening [12]. Therefore, one is less likely to accept opposing ideas. When arousal is controlled, valence determines the recall of information to the degree that positive messages are remembered better [13], which possibly enhances the self-affirmation of confirming evidence in decisionmaking and introduces the other two constructs: valence and working memory. Tying back to previous discussions on traits vs. states, a "state" like response during confirmation bias can be partially defined as a momentary emotional reaction to internal and external trigger since the duration and intensity of the emotion experienced are contingent on various factors, and once the emotional reaction passes, a state of equilibrium resumes. Thus, although both approaches to understanding confirmation bias, trait/state and cognitive/emotional, can exist in different ways, the brain tends to abandon respect for this distinction and mix the two perspectives to construct an architecture of factors that promote confirmation bias.

Table 1. Definitions

\begin{tabular}{|l|l|}
\hline $\begin{array}{l}\text { Confirmatio } \\
\text { n Bias }\end{array}$ & $\begin{array}{l}\text { A psychological tendency to seek } \\
\text { confirmatory evidence and ignore opposing } \\
\text { information. }\end{array}$ \\
\hline Uncertainty & $\begin{array}{l}\text { The state of doubt towards the future or } \\
\text { about what is the appropriate thing to do. }\end{array}$ \\
\hline $\begin{array}{l}\text { Valence (in } \\
\text { emotion) }\end{array}$ & $\begin{array}{l}\text { The extent to which an emotion is positive or } \\
\text { negative that a stimulus generates. }\end{array}$ \\
\hline $\begin{array}{l}\text { Working } \\
\text { memory }\end{array}$ & $\begin{array}{l}\text { A cognitive system in which human beings } \\
\text { encode, organize and extract information } \\
\text { from, often used for rational reasoning. }\end{array}$ \\
\hline $\begin{array}{l}\text { Positivity } \\
\text { bias/prefere } \\
\text { nce }\end{array}$ & $\begin{array}{l}\text { The priority for pleasantness when } \\
\text { observing and experiencing events. }\end{array}$ \\
\hline $\begin{array}{l}\text { Negativity } \\
\text { bias }\end{array}$ & $\begin{array}{l}\text { The greater influence on people's } \\
\text { psychological state by things of a more } \\
\text { negative nature. }\end{array}$ \\
\hline $\begin{array}{l}\text { Information } \\
\text { Overload }\end{array}$ & $\begin{array}{l}\text { The difficulty in understanding a context or } \\
\text { an issue and decision-making when facing } \\
\text { overwhelming information. }\end{array}$ \\
\hline $\begin{array}{l}\text { Information } \\
\text { utility }\end{array}$ & $\begin{array}{l}\text { The value of a product that is determined by } \\
\text { how much useful information it could } \\
\text { provide. }\end{array}$ \\
\hline
\end{tabular}

\section{FACTORS TO CONSIDER}

\subsection{Uncertainty}

Throughout human history, information has been an indispensable factor for survival. Just like the saying "Know your enemy" by Sun Tzu, human ancestors acquired information about other species and their environments to forage, avoid becoming prey, and prevent, more generally, uncertainty about their surroundings. Today's many incidents also require confronting the unknown and making difficult decisions, especially in pernicious situations where catastrophic phenomena and epidemic diseases like COVID-19 occur. However, the paucity of information, or uncertainty, is a state of lacking necessary knowledge on certain situations, restricting one's judgments in a limited range of possible guesses, often causing stress and inducing aversive affect. Economist Frank Knight [14] further added to the definition of uncertainty by drawing the fundamental difference between uncertainty and risk, a situation where a specific probability is assigned to every outcome. He defined it as an immeasurable situation of unknown probabilities impossible to calculate.

Uncertainty exemplifies itself almost everywhere. For example, in clinical conditions, intolerance of uncertainty is found to be one of the most commonly observed risk factors associated with patients suffering from Generalized Anxiety Disorder (GAD), ObsessiveCompulsive Disorder (OCD), and Social Anxiety Disorder (SAD) [15]. Many symptoms of these anxiety disorders, such as worrying and avoiding social contact, can be understood as attempts to reduce one's uncertainty such that worry enables one to envision the future and evaluate potential risks. At the same time, avoidance allows limited exposure to unknown stimuli. From a biological approach, unpredictability incites the amygdala's response to non-biologically relevant stimuli when they occur unpredictably, while also driving people to adjust their behavioral and learning policies depending on its different levels [16]. These reactive responses can be interpreted as evidence for people's strategic attempts to deal with their lack of information and, therefore, confidence in decision-making, which introduces the thrust of this particular section: the possibility of uncertainty's causal existence in the formation of confirmation bias.

Much evidence suggests that confirmation bias arises robustly in acquiring and utilizing information. As prior studies posit confirmation bias as one of the predominant causes for the proliferation of misinformation, it is feasible that people choose confirmatory evidence in an unconscious attempt to reduce their uncertainty about perplexing situations. For instance, during the times of COVID-19, information overload was discovered to be a reinforcement for the spread of cyberchondria on unverified information sharing [17], underlining people's 
unpreparedness to the cascade of information and their need to avoid uncertainty. In addition to the emergence of uncertainty, the total absence of uncertainty, or one's confidence in one's belief and judgment in a given context, is also relevant to confirmation bias. A study combining human magnetoencephalography (MEG) with behavioral and neural modeling demonstrated that people with high confidence often develop an alternation in their post-decision neural processing. The integration of supportive evidence is augmented, and the opposing is obliterated [18]. In other words, confidence seems to drive a selective neural modulation of confirmation bias after decision-making, and the extremities on either side --- whether having abundant uncertainty or none --essentially render people to develop according to mechanisms, converging to the same informationprocessing heuristic.

Overall, several findings suggest that uncertainty is arguably salient to most people, perhaps because of its evolutionary role in human survival. Indeed, clinical populations show a dysfunctional mode of dealing with uncertainty that implies the need to accurately, or at the very least, gather information to make predictions. The author proposes that uncertainty facilitates and amplifies the occurrence of confirmation bias, as people have limited capacity for information-processing and therefore yield to the available information, which often revolves around information that has been previously learned. When dealing with uncertainty, decision-makers tend to form different hypotheses. They are evaluated by "considering the conditions under which the hypothetical event is expected to occur (to see if it happens) or by examining known instances of its occurrence" [5], and ultimately choosing the hypothesis that is more likely to produce a positive experience than the correct outcome. Such a process is even more frequent and influential when the incorrect hypothesis possesses significant similarities to one's pre-existing beliefs. The human brain is susceptible to being positively reinforced by the "positivity" of the chosen hypothesis, thereby preceding the search for further possibilities and accepting disconfirmatory evidence. An incorrect hypothesis is significantly similar to the correct one when it resembles the majority of its contents with only a diminutive discrepancy. By way of illustration, if an individual holding negative attitudes toward politicians searches for political news, one tends to develop hypotheses on the content of the news based on their titles and sources of publications with the one's previous knowledge on passages of the same kind. After a process of deduction and evaluation, he would disregard articles of positive appraisal on politicians that hardly fit his previous expectations and enjoy reading critical articles that resemble much of his own opinions. Repeated occurrences of a situation like this gradually reinforce the human brain to become accustomed to confirmatory information only since people repeatedly receive positive feedback from their decisions. Consequently, people quit seeking further choices and information, just as the individual in the previous example would eventually ignore opposing articles and no longer value the variety of information sources. It may be fundamentally difficult for human beings to withstand uncertainty, and, therefore, people are always in the search for ways to eradicate the notion that "people are unsure." As discussed earlier in this section, an evolutionary cause possibly exists behind this phenomenon. Uncertainty drives us to develop heuristics to digest information and make decisions in a quick, efficient fashion so that the survival of our species is guaranteed. It provides the cause for the emergence of confirmation bias. Also, it allows another construct, namely valence, to play an important role in the manifestation of confirmation bias. The lack of certainty elicits negative feelings, such as fear, as will be detailed in the next section.

\subsection{Valence}

In affective science--the study of emotion--valence is defined as a spectrum that describes positive states existing on one end and negative states on the other side. Emotion categories such as fear, sadness, and anger are typically considered negative emotional states, or in other words, negatively valenced emotional states. In contrast, emotion categories such as pride, happiness, and relief are typically considered positive emotional states, or in other words, positively valenced emotional states. With its universal presence behind many psychological phenomena (e.g., voting behavior; criminal investigation), valence may be a contributing or moderating effect of confirmation bias. One recent study showed that counter-attitudinal information could influence a pre-existing attitude and the associated confirmation bias [19]. In the present context, confirmation bias is discussed as having relationships with valence in the following two scenarios: in a method whereby people maintain positive affect or a negative outcome resulting from people's general unwillingness to be challenged, particularly with strongly held beliefs.

In the first case, confirmation bias emerges from motivated attempts to obtain emotional satisfaction like confidence and gratification and even competence over how the world functions [20]. Human beings typically have a natural inclination to stay in a positive mood, which drives them to develop a preferential attitude towards pleasant thoughts and memories over displeasing ones. As a result, beliefs and the intake of evidence during decision-making are both susceptible to emotion. For example, when an individual faces two options, it is quite sensible to discard opposing data and selectively lay more weight on evidence that fits their expectations. In this case, cognitive bias arises as a way for people to preserve positive effects, either consciously or unconsciously, an effect coined as "the Pollyanna 
principle" of confirmation bias [5]. Another possible explanation for pleasure maintenance is humanity's inferential strategies, stating that they are adapted to identify potential rewards and the avoidance of costly errors rather than the science of reason and logic. It is viewed as a practice of pragmatism because people solve problems practically instead of utilizing fixed ideas and theories [5]. While pragmatism produces some useful results, it inherently weakens the objectivity of decisionmaking. After all, it shifts people's positions toward those choices that offer the greatest rewards, satisfying their emotional needs but doing little to solve the real problem because it incites confirmation bias.

On the other hand, confirmation bias allows one to maintain a pleasant mood and prevents one from experiencing negative valence by avoiding psychological damage (e.g., events like uncertainty). Based on the appraisal theory of emotions, which defines emotions as the process of adapting to the survival and reproduction of life by assessing stimuli in the environment, uncertainty can be seen as an assessment dimension on which the rise in valence depends. For instance, the negatively valenced affect "disappointment" may be related to the certainty about a positive result such as reward-seeking or decision-making. When an individual holds the staunch belief in his opportunity for winning a prize or making the right decision, he shall experience a greater degree of disappointment than one foresees pessimistically about his chances. Thus, the perceived uncertainty about a situation can be a fundamental determinant of a specific emotional process. On this basis, it has been noted that uncertainty can have effects that can seriously disrupt people's lives in general. This is because one study showed that people diagnosed with anxiety or depression scored significantly higher on intolerance of uncertainty than those who had never suffered from such disorders, highlighting the substantial role that uncertainty plays as a cross-diagnostic factor in emotional disorders. [21]. Moreover, Carleton also posits that fear of the unknown fundamentally underlines all kinds of fear in life. This theoretical proposition, along with the evidence presented above, suggested a critical role of uncertainty in negative valence. Furthermore, viewing disconfirming evidence also imposes a threat to people's decisions, which evokes negatively valenced emotions for facing their failure and causes uncomfortable "psychological loss" such as embarrassment and disappointment [22]. Thus, driven by their propensity for pleasure and comfort, people strive to mitigate this aversion since negative valence requires more of people's emotion regulation skills than positive valence. Simply confirming the current evidence seems to be a much easier and salient solution than trying to find ways to regulate negative emotions. In other words, similar to how patients with anxiety utilize worry to regulate uncertainty, individuals -- clinical or not -- turn to confirmation bias for regulating negative valence linked with uncertainty.

Overall, both the motivation to maintain positive effects and avoid negative effects drives the development of confirmation bias in the mass of information. This is arguably a philosophical argument that there is a dilemma for the human mind - that is, the brain - which is almost imprisoned by confirmation bias because in either direction - positive or negative - the heuristic seems to help. It also raises the question of whether human mental life is biased in areas such as memory. To answer such a question, the next section describes the relationship between confirmation bias and working memory.

\subsection{Working Memory}

The concept of working memory has been diffusely studied since the 1960s, stemming from cognitive psychology to many facets such as cognitive science and neuroscience. It is usually defined as a brain system or set of systems enabling the "temporary storage of information" relating to various performances of complex cognitive tasks such as language comprehension, decision-making, and reasoning [23]. For example, the ability to hold a cell phone number in mind or remember foreign phrases over a brief period before they transform into long-term memory are practical uses of working memory in everyday life. The author specifically chooses to focus on working memory instead of short-term or long-term memory because it is more in tune with the immediate context, arguably where confirmation bias often unfolds [5]. It is also noteworthy that when bringing working memory into the discussion, the author specifically relates working memory to the reasoning of affective experience, as in, for example, reasoning through negative valence brought on by uncertainty. As information flows from the environment, people attend to different stimuli for selection and later processing, indicating an inevitably crucial relationship between working memory and confirmation bias. One study has demonstrated that recognition memory performance for information that is supportive of the participants' viewpoint is better than that for opposing information [24], which proposes the possible idea that confirmation bias enhances working memory.

In this paper, the author suggests a bidirectional relationship between working memory and confirmation bias. In decision-making, working memory necessitates confirmation bias, which reinforces the memorization of certain evidence over others. Nickerson [5] introduced the idea of a conditional reference frame, delineating how one specific hypothesis can lead to the establishment of a focal hypothesis assumed to be true and, in turn, influence subsequent $\mathrm{j}$ judgments. Essentially, an individual can never escape the boundaries from a hypothesis once he determines one at the beginning of the 
decision-making process, which implies that the limited capacity of attention and working memory exert a significant influence on confirmation bias. On the other hand, individuals who experience confirmation bias also face potential memory alteration. For example, a recent study showed that members of juries who held preexisting biases towards the defendant exhibited noticeable differences at taking notes and making judgments from those with no such biases [25]. Therefore, it is obvious that working memory and confirmation bias share a mutual stimulation, leading the decision-making process to extremity.

\section{AN ARCHITECTURE}

In addition to outlining the factors of confirmation bias, the present paper attempts to delineate how confirmation bias--as a phenomenon and mechanism-manifests itself both behaviorally and psychologically. In this vein, the author draws on insights from social and cognitive psychology, neuroscience, and affective science to offer a theory-driven mental representation of confirmation bias.

As shown in Figure 1, uncertainty is considered the primary catalyst for confirmation bias between the proposed factors since it habitually propels the human tendency to seek information. In other words, the degree to which humans understand their immediate contexts is predicated on their ability to make sense of them, whether that is through critical thinking, heuristics, or, simply, biases such as cognitive biases. Moreover, uncertainty leads to at least two different responses: a reaction (e.g., emotion through valence) and a means to regulate that reaction (e.g., reasoning through working memory cognitive components). Here, the author will first discuss the relationship between each of these factors then dive into their roles in manifesting confirmation bias.

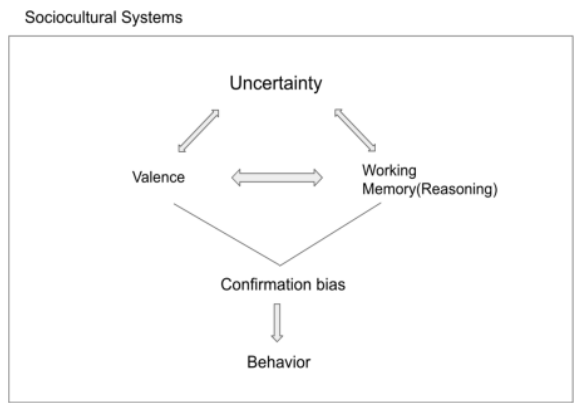

Figure 1 Model Representation of Confirmation Bias

\subsection{Uncertainty and Valence}

Among various theories regarding valence, the appraisal theories appear to be particularly relevant for the present discussion, for they consider emotions as directly resulting from the perceived certainty of a situation along with other appraisal evaluations (for a review on appraisal theories, see [26]). How one perceives the probable outcomes of an ongoing event -whether it evokes positive or negative effects -- can significantly influence the individual's subsequent action. For example, in interviewing for new jobs, if the likelihood of acceptance is high, the interviewee often feels positively valenced effects such as joy and excitement, anticipating positive long-term after-effects like receiving satisfactory payment, gaining wealth, and purchasing desired items. The interviewee might also cancel other interviews because the perceived chance of success is felt as certain. On the other hand, If the likelihood is perceived as low, the interviewee is likely to feel negative, such as sadness, frustration, or worry, experiencing negative long-term after-effects. The individual might react by seeking more opportunities or giving up the search entirely. Therefore, it is reasonable to conclude that anticipation of future events, based on the relevant probability of an outcome, determines emotions after perceiving the degree of uncertainty in a specific way.

While uncertainty can drive both positive and negative effects, the inverse relationship is discussed in several studies. One study has shown that effect alters perceptions of the risk of uncertain events by purporting the evidence that reading a newspaper story about a tragic death causes participants to overrate the probability of future fatal events by 74\% [27]. Moreover, specific emotions also change people's likelihood to interpret subsequent events as consistent with how they are feeling. For example, if a person were to feel sad, they would interpret the likelihood of subsequent events more pessimistically [28]. As a result, uncertainty influences the positivity or negativity associated with events, whereas the resulting valence alters how an individual anticipates subsequent events.

\subsection{Uncertainty and Working Memory}

In general, approaching uncertainty with a problemsolving mindset bodes well for people, but doing so is predicated on using working memory. Another approach proposed in the configuration demonstrates the following way. While current accounts for the observed biases indicate the limited human capacity to process the entire set of available information, making it difficult to investigate the unknown in a neutral, scientific way, researchers excavate to understand the working memory mechanism. Due to its ubiquitous presence, it is unsurprising that a connection between it and the fundamental construct for all, uncertainty, has gradually emerged. In this paper, the author believes the two constructs share a bidirectional relationship in which one serves to reduce another and vice versa.

According to relevant evidence, working memory representations tend to prioritize the memory-matching items at a post-perceptual level by regarding a target's 
location without altering its perceptibility, therefore alleviating uncertainty during visual research. Researches have not only demonstrated the power of working memory in reducing uncertainty but have also shown that in the face of uncertainty requiring cognitive resources. NFC (Need for closure) mediates the effect of BIS (Behavioral Inhibitory System) on behavior in a decision-making situation; working memory capacity was thought to moderate this relationship. For example, as obsessive-compulsive disorder (OCD) is known to be associated with visuospatial working memory deficits [29], intolerance of uncertainty is also considered to be an essential ingredient of OCD symptoms. Current research results support a correlation between the two constructs, as trials with greater uncertainty were found to show a decrease in visuospatial working memory efficiency to $65 \%$ of usual performance in OCD patients, but not in healthy subjects [30]. Essentially, deficient working memory performance correlates with the predisposition to experience uncertainty in healthy subjects. In contrast, uncertainty contributes to a decline in the persistent performance of working memory in OCD patients, presenting an inverse temporal relationship. To sum up, working memory offers human beings an efficient mechanism to manage uncertainty while possibly receiving some backfires in return, both as a way of encoding and a gate for extracting information.

\subsection{Valence and Working Memory}

Both valence and working memory are driven by the need to resolve uncertainty and have a complex relationship with each other. Though it is widely acknowledged that events involving strong emotions lead to a high degree of memory vividness [31], the valence of those events often come with conflicting results. On the one hand, positive affect enhances verbal and spatial working memory with more successful executive control, whereas negative affect does not. In one study, people performed significantly better in a memory task when the trials were paired with happy facial expressions than not [32]. On the other hand, there is a negativity bias in working memory, such as a superior recall of threatening faces in the visual search paradigm [33].

Utilizing various activation topographies on cortical and deep brain structures during memory retention with different valence, researchers discovered negative emotional valence of information. When combined with levels of emotional arousal, it interferes with the formation of optimal functional brain systems, thereby decreasing working memory retention efficiency [34]. Clinical approaches also came into play when it was found that patients with schizophrenia tended to perform less well than healthy subjects in terms of working memory accuracy [35]. The combination of the above evidence highlights the importance of integrating neural, clinical, and behavioral levels of analysis and proves the relationship between valence and working memory to be a multi-disciplinary mechanism that requires further research.

\subsection{On Confirmation Bias}

After analyzing the relationships between the three constructs -- uncertainty, valence, and working memory, the author proceeds to analyze their overall influence on confirmation bias from two approaches, emotion and reasoning, which play an inter-related role on the heuristic. According to the risk-as-feelings theory, people use emotions to make decisions in risky situations that diverge from and precede cognitive evaluations to drive behaviors [36], providing evidence for these two approaches that uncertainty incites and exert influence on. The author proposes that people emphasize confirming over disconfirming evidence because they tend to pursue pleasant emotions and use conceited reasoning to reduce uncertainty about ongoing events, and both valence and working memory help human beings to accomplish such goals. In the configuration, the author believes that in decision-making, an individual prefers the use of reasoning for self-righteousness and a feeling of justification. They conceal, either unconsciously or consciously, the fact that one's experiences on positive or negative effects lead oneself to suppress opposite evidence or amplify supportive ones, essentially overthrowing the definition of rational thinking. In other words, humans rely on working memory to make seemingly rational decisions. At the same time, valence has already encroached on that mechanism, causing the relative cognitive process and subsequent behaviors to experience a significant deviation from perfect rationality.

\section{DISCUSSION}

In this paper, the author first introduced an understanding of confirmation bias based on two approaches, state vs. trait and affective vs. cognitive, and three relevant constructs -- uncertainty, valence, and working memory -- to generate a configuration of the bias' manifestation. The author discussed each of the factors and analyzed them concerning confirmation bias. When it comes to uncertainty, the limited capacity of attention is indeed relevant to confirmation bias, as the human brain is only designed for a finite amount of processing at a given time. To reduce confirmation bias and improve decision-making, more attention should be paid to improving critical thinking skills. During the attention assignment phrases, a decision-maker often prefers strong signals that suggest favorable outcomes for processing [37]. However, it is likely that confirmation bias, particularly in a self-relevant context, disrupts, at the very least momentarily, emotionality. It is also likely that the affective component of confirmation bias produces more "cognitive" features, such as discounting disconfirming evidence [5], or that other cognitive 
function plays an essential role in manifesting confirmation bias, such as remembering only confirming evidence in a given context [24]. After analyzing the three constructs, the author explained the configuration as a whole, and then elaborated on the inter-related relationship between the constructs and their overall influence on confirmation bias. The author proposes that emotion and reasoning, accompanied by working memory, are the two approaches induced by unknown stimuli to motivate confirmation bias to reduce uncertainty, which in turn drives the observed behaviors of discrediting opposite evidence and valuing supportive ones. Like most other biases, confirmation bias is a mixed product of effects and cognition for responding to the unknown on the path of evolution.

\section{IMPLICATION}

As a ubiquitous phenomenon worldwide, confirmation bias exerts its influence not only in people's daily lives but also in circumstances like criminal investigation and online political discussion. Confirmation bias arises at different stages of the criminal procedure and plays a pivotal role in influencing the administration of justice. In some cases, police officers under emotional circumstances, such as anger or grief, may replace empirical evidence with confirmation bias "as a catalyst for decision making" in the investigative process [38]. This is an example of the influence that overrides rational thinking and leads to the confirmation bias presented in the model. Not only in criminal investigations, confirmation bias also occurs in judicial decisions, such as when judges' perceptions of the strength of presented evidence are susceptible to pretrial detention, making the cases of suspects in their custody more likely to result in convictions [39]. Moreover, according to another study by the same researchers, prosecutors demonstrate confirmation bias after deciding to press charges by being less willing to agree on extra investigation and proposing more guiltconfirming investigation [39]. Therefore, people, especially those participating in the judicial systems, should pay extra attention to the possible occurrence of confirmation bias, preventing it from damaging innocent people and distorting the practice of justice.

Another frequent appearance of confirmation bias is among online users who express their attitudes and opinions almost limitlessly. As mentioned in the previous discussion, data analysis on personal preference creates filters and other kinds of selective exposure of information to restrain users only in what they accept and feel accepted. They select statements that adhere to their beliefs and never notice the dissenting ones, leading to group polarization, prohibiting people with different interests from exchanging ideas, and reducing the diversity of the internet community. This phenomenon is especially prevalent in politics, as one of the most controversial and significant topics nowadays. A study has shown that selective exposure, regardless of source quality, reinforced political attitudes and brought out more extreme political polarization [40].

\section{OUTSTANDING QUESTIONS}

The first question concerns the occasional preference towards unknown stimuli shown in most human beings. For example, a birthday surprise is often welcome in most cultures, and the Disneyland Haunted mansion remains popular over years of visits. Considering the dislike of uncertainty highlighted in this paper, it seems contradictory and confusing that some people enjoy the mysterious atmosphere and a sense of the unknown towards the surrounding environment. Therefore, the rise of confirmation bias in the face of such preference seems quite worthy of further research. If people hate uncertainty, why do many people enjoy visiting haunted houses and watching horror movies? If people somehow prefer uncertainty, why would heuristics like confirmation bias be developed to help people minimize uncertainty? Although the present paper lacks empirical evidence, one explanation is that people need security to stay alive and curiosity to incite novel developments on the path of evolution. With an appropriate and preferably controllable amount of uncertainty about the world, human beings are more motivated to gather information and dig for the reasons behind things, increasing survival rates and ensuring a greater possibility of significant progress. Hence, uncertainty can be both fascinating and intimidating to humans. In short, further studies are therefore encouraged to offer additional insight, investigating the exact role confirmation bias and uncertainty play in the process of decision-making.

The second question arises between the conflicting existence of positivity and negativity bias and where confirmation bias fits into the gradient. While it is most reasonable and natural for human beings to pursue a positive aspect of reality, such as a delicious meal or compliments from others, there are occasions when the tendency to replace the emphasis on negative stimuli has a greater impact on people. There seems to be a spectrum with the best outcome of things at one end and the worst at the other, which raises the question of where confirmation bias exists on such a gradient. At first glance, it appears to be more associated with the positive side, as it tends to overrate supportive evidence and experience a pleasant mood. Still, the need to reduce uncertainty is also associated with an emphasis on the negative side of the world. Thus, more research is needed to investigate the role of confirmation bias with positivity and negativity bias.

The relationship between valence and confirmation bias brings up a final question, which primarily focuses on the afterword influence of the latter. Despite the discussion of how valence influences, or even determines 
the manifestation of confirmation bias, there is still no concrete evidence as to whether people who exhibit confirmation bias indeed experience a positive mood. Therefore, the author proposes that future research should investigate the relationship between the emergence of confirmation bias and the valence of its after-effects, which may be positive, providing more evidence for the correlation between emotion and confirmation bias, or even other kinds of cognitive bias in general.

\section{CONCLUSION}

This paper not only reviews past and present studies on confirmation bias, but discusses three basic constructs concerning the bias and each other to eventually proposes a mechanism for understanding confirmation bias in a relatively new way. The significance of the paper arises from its summary of a considerable amount of relevant studies on the phenomenon, its attempt to consider uncertainty as the major reason behind confirmation bias, and its analysis on which the bias manifests itself through valence and working memory. Future studies are recommended to extend the analysis into studying the more prolonged consequence of confirmation bias and focus on investigating the contextual factors underlying each instance of confirmation bias, especially the role of social media in manifesting the bias. With the popularization and rapid development of social media providing a broad platform for the intake and exchange of information, data analysis on personal preference creates filters and other kinds of selective exposure of information to restrain users only in what they accept and feel accepted. Therefore, confirmation bias under such circumstances might evolve into group polarization, prohibiting people with different interests from exchanging ideas and reducing the diversity of the human population. The author suggests future research to pay close attention to the issue, designing specific studies to measure the possible relationship between confirmation bias, attitude polarization, and social media usage.

With its ability to distort the value of information through emotion and cognitive thinking, confirmation bias can indeed be an obstacle to the pursuit of truth to some extent. However, confirmation bias can also be interpreted as rational. It is an attempt to ensure that people's thinking, or even personality, is consistent and stable so that people are not shattered with the continuous upcoming of new evidence. After all, opinions are never isolated but followed by corresponding patterns of behavior and countermeasures. In many cases, what people need most is not the "truth" but a set of tried and tested methods to guide people on how to live their lives, and there is no doubt confirmation bias succeeds in this regard.

\section{REFERENCES}

[1] B.B. Doll, K.E. Hutchison, M.J. Frank, Dopaminergic Genes Predict Individual Differences in Susceptibility to Confirmation Bias, in: Journal of Neuroscience, 31(16), 2011, pp. 6188-6198. DOI: https://doi.org/10.1523/JNEUROSCI.6486-10.2011

[2] D. Buttelmann, N. Zmyj, M. Daum, M. Carpenter, Selective Imitation of In-Group Over Out-Group Members in 14-Month-Old Infants, in: Child Development, 84(2), 2013, pp. 422-428. DOI: https://doi.org/10.1111/j.1467-8624.2012.01860.x

[3] J. Wikstrom, L.G. Lundh, J. Westerlund, L. Högman, Preattentive bias for snake words in snake phobia? In: Behaviour Research and Therapy, 42(8), 2004, pp. 949-970. DOI: https://doi.org/10.1016/j.brat.2003.07.002

[4] J. Klayman, Varieties of Confirmation Bias, in: Psychology of Learning and Motivation, 32, 1995, pp. 385-418. DOI: https://doi.org/10.1016/S00797421(08)60315-1

[5] R.S. Nickerson, Confirmation bias: A ubiquitous phenomenon in many guises, in: Review of General Psychology, 2(2), 1998, pp. 175-220. DOI: https://doi.org/10.1037/1089-2680.2.2.175

[6] C.G. Lord, L. Ross, M.R. Lepper, Biased assimilation and attitude polarization: The effects of prior theories on subsequently considered evidence, in: Journal of Personality and Social Psychology, 37(11), 1979, pp. 2098-2109. DOI: https://doi.org/10.1037/0022-3514.37.11.2098

[7] G.W. Allport, H.S. Odbert, Trait-names: A psycholexical study, in: Psychological Monographs, 47(1), 1936, pp. i-171. DOI: https://doi.org/10.1037/h0093360

[8] J.R. Nesselroade, T.W. Bartsch, Multivariate perspectives on the construct validity of the traitstate distinction, in R. B. Cattell \& R. M. Dreger (Eds.), Handbook of modern personality theory, 1977, pp. 221-238. Washington, DC: Hemisphere.

[9] A. Kappes, A.H. Harvey, T. Lohrenz, P.R. Montague, T. Sharot, Confirmation bias in the utilization of others' opinion strength, in: Nat Neurosci 23, 2020, pp. 130-137. DOI: https://doi.org/10.1038/s41593-019-0549-2

[10] S. Knobloch-Westerwick, S.B. Kleinman, Preelection Selective Exposure: Confirmation Bias Versus Informational Utility, in: Sage Journals, 39(2), 2012, pp. 170-193. DOI: https://doi.org/10.1177/0093650211400597 
[11] K.H. Kim, A. Cho, Ego Identity Status and Confirmation Bias in Career Information Search, in: Journal of Asia Pacific Counseling, 10(1), 2012, pp. 187-208. DOI : https://doi.org/10.18401/2012.2.2.5

[12] C. MacLeod, I.L. Cohen, Anxiety and the interpretation of ambiguity: A text comprehension study, in: Journal of Abnormal Psychology, 102(2), 1993, pp. 238-247. DOI: https://doi.org/10.1037/0021-843X.102.2.238

[13] A. Lang, K. Dhillion, Q. Dong, The effects of emotional arousal and valence on television viewers' cognitive capacity and memory, in: Journal of Broadcasting \& Electronic Media, 39(3), 1995, pp. 313-327. DOI: https://doi.org/10.1080/08838159509364309

[14] F.H. Knight, Risk, Uncertainty, and Profit, Boston: Hart, Schaffner \& Marx, 1921.

[15] P.A. Boelen, A. Reijntjes, Intolerance of uncertainty and social anxiety, in: Journal of Anxiety Disorders, 23(1), 2009, pp. 130-135. DOI: https://doi.org/10.1016/j.janxdis.2008.04.007

[16] I.E. Monosov, How Outcome Uncertainty Mediates Attention, Learning, and Decision-Making, in: Trends in Neurosciences, 43(10), 2020, pp. 795-809. DOI: https://doi.org/10.1016/j.tins.2020.06.009

[17] S. Laato, A.K.M.N. Islam, M.N. Islam, E. Whelan, What drives unverified information sharing and cyberchondria during the COVID-19 pandemic? in: European Journal of Information Systems, 29(3), 2020, pp. 288-305. DOI: https://doi.org/10.1080/0960085X.2020.1770632

[18] M. Rollwage, A. Loosen, T.U. Hauser, R. Moran, R.J. Dolan, S.M. Fleming, Confidence drives a neural confirmation bias, in: Nat Commun, 11, 2020, pp. 2634. DOI: https://doi.org/10.1038/s41467-020$16278-6$

[19] P. Dibbets, C. Meesters, Disconfirmation of confirmation bias: the influence of counterattitudinal information, in: Curr Psychol, 2020. DOI: https://doi.org/10.1007/s12144-020-00744-X

[20] B.J. Casad, Confirmation bias, in: Encyclopedia Britannica, 2019. https://www.britannica.com/science/confirmationbias

[21] R.N. Carleton, The intolerance of uncertainty construct in the context of anxiety disorders: theoretical and practical perspectives, in: Expert Rev Neurother, 12(8), 2012, pp. 937-947. DOI: https://doi.org/10.1586/ern.12.82
[22] J.L. L. Thorogood, M.T.. T. Crichton, A.. Bahamondes, Case Study of Weak Signals and Confirmation Bias in Drilling Operations, in: SPE Drill \& Compl, 29, 2014, pp. 304-310. DOI: https://doi.org/10.2118/168047-PA

[23] A. Baddeley, Working memory, in: Current Biology, 20(4), 2012, R136-R140. DOI: https://doi.org/10.1016/j.cub.2009.12.014

[24] P. Frost, B. Casey, K. Griffin, L. Raymundo, C. Farrell, R. Carrigan, The Influence of Confirmation Bias on Memory and Source Monitoring, in: The Journal of General Psychology, 142(4), 2015, pp. 238-252.

DOI: https://doi.org/10.1080/00221309.2015.1084987

[25] S.M. Kassin, L.S. Wrightsman, The American Jury On Trial: Psychological Perspectives, Hemisphere Publishing Corporation, 2013.

[26] K.R. Scherer, Appraisal theory, in: T. Dalgleish \& M. J. Power (Eds.), Handbook of cognition and emotion, John Wiley \& Sons Ltd, 1999, pp. 637 663.

DOI: https://doi.org/10.1002/0470013494.ch30

[27] E.J. Johnson, A. Tversky, Affect, generalization, and the perception of risk, in: Journal of Personality and Social Psychology, 45(1), 1983, pp. 20-31. DOI: https://doi.org/10.1037/0022-3514.45.1.20

[28] D. DeSteno, R.E. Petty, D.T. Wegener, D.D. Rucker, Beyond valence in the perception of likelihood: The role of emotion specificity, in: Journal of Personality and Social Psychology, 78(3), 2000, pp. 397-416. DOI: https://doi.org/10.1037/0022-3514.78.3.397

[29] N.J. van der Wee, N.F. Ramsey, J.M. Jansma, et al., Spatial working memory deficits in obsessive compulsive disorder are associated with excessive engagement of the medial frontal cortex, in: NeuroImage, 20(4), 2003, pp. 2271-2280. DOI: https://doi.org/10.1016/j.neuroimage.2003.05.001

[30] V. Lambrecq, J. Rotge, N. Jaafari, et al., Differential role of visuospatial working memory in the propensity toward uncertainty in patients with obsessive-compulsive disorder and in healthy subjects, in: Psychological Medicine, 44(10), 2012, pp. 2113-2124. DOI: https://doi.org/10.1017/S0033291713002730

[31] B.R. Lindström, G. Bohlin, Emotion processing facilitates working memory performance, in: Cognition and Emotion, 25(7), 2011, pp. 1196-1204. DOI:

https://doi.org/10.1080/02699931.2010.527703 
[32] A.A. Gonzalez-Garrido, A.L. López-Franco, F.R. Gómez-Velázquez, J. Ramos-Loyo, H. Sequeira, Emotional content of stimuli improves visuospatial working memory, in: Neuroscience Letters, 585, 2015, pp. 43-47. DOI: https://doi.org/10.1016/j.neulet.2014.11.014.

[33] C.H. Hansen, R.D. Hansen, Finding the face in the crowd: An anger superiority effect, in: Journal of Personality and Social Psychology, 54(6), 1988, pp. 917-924. DOI: https://doi.org/10.1037/00223514.54.6.917

[34] R. Rozovskaya, E. Pechenkova, E. Mershina, R. Machinskaya, fMRI Study of Retention of Images with Different Emotional Valence in the Working Memory, in: Journal of the Higher School of Economics, 11(1), 2014, pp. 27-48.

[35] S. Park, P.S. Holzman, P.S. Goldman-Rakic, Spatial Working Memory Deficits in the Relatives of Schizophrenic Patients, in: Arch Gen Psychiatry, 52(10), 1995, pp. 821-828. DOI: https://doi.org/10.1001/archpsyc.1995.0395022003 1007

[36] G.F. Loewenstein, E.U. Weber, C.K. Hsee, \& N. Welch, Risk as feelings, in: Psychological Bulletin, 127(2), 2001, pp. 267-286. DOI: https://doi.org/10.1037/0033-2909.127.2.267

[37] B.T.K. Leung, Limited cognitive ability and selective information processing, in: Games and Economic Behavior, 120, 2000, pp. 345-369. DOI: https://doi.org/10.1016/j.geb.2020.01.005

[38] W.A. Wallace, The Effect of Confirmation Bias on Criminal Investigative Decision Making, Walden Dissertations and Doctoral Studies, 407, 2015.

[39] M. Lidén, Confirmation Bias in Criminal Cases, Uppsala University Publications, 2018.

[40] A. Westerwick, B.K. Johnson, S. KnoblochWesterwick, Confirmation biases in selective exposure to political online information: Source bias vs. content bias, Communication Monographs, 84(3), 2017, pp. 343-364. DOI: https://doi.org/10.1080/03637751.2016.1272761 